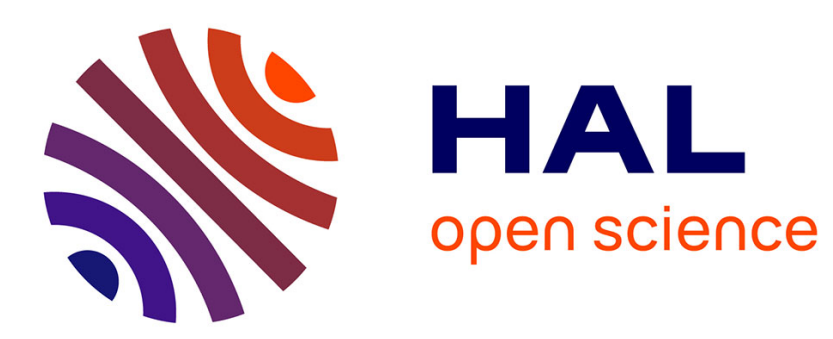

\title{
Calcul semi-tridimensionnel des champs et des courants dans les machines cylindriques asynchrones sans fer
}

\author{
F. Rioux-Damidau
}

\section{To cite this version:}

F. Rioux-Damidau. Calcul semi-tridimensionnel des champs et des courants dans les machines cylindriques asynchrones sans fer. Revue de Physique Appliquée, 1983, 18 (2), pp.113-127. 10.1051/rphysap:01983001802011300 . jpa-00245071

\section{HAL Id: jpa-00245071 https://hal.science/jpa-00245071}

Submitted on 1 Jan 1983

HAL is a multi-disciplinary open access archive for the deposit and dissemination of scientific research documents, whether they are published or not. The documents may come from teaching and research institutions in France or abroad, or from public or private research centers.
L'archive ouverte pluridisciplinaire HAL, est destinée au dépôt et à la diffusion de documents scientifiques de niveau recherche, publiés ou non, émanant des établissements d'enseignement et de recherche français ou étrangers, des laboratoires publics ou privés. 


\title{
Calcul semi-tridimensionnel des champs et des courants dans les machines cylindriques asynchrones sans fer
}

\author{
F. Rioux-Damidau \\ Laboratoire d'Electrotechnique des Universités Paris-VI et XI (*), Bâtiment 214, 91405 Orsay, France
}

(Reçu le 24 mai 1982, révisé le 10 novembre, accepté le 15 novembre 1982)

\begin{abstract}
Résumé. - On effectue la décomposition en série de Fourier spatiale (en $z$ et $\theta$ ) et temporelle des champs magnétiques et courants existant dans une machine cylindrique asynchrone sans fer en considérant que le rotor est infiniment long, mais que le stator a sa structure réelle, de longueur finie. Les expressions analytiques obtenues pour les champs sont très faciles à programmer et permettent de calculer les caractéristiques.

On donne entre autres deux expressions permettant le calcul de la puissance active par intégration des champs : au niveau de la surface du rotor pour l'une, dans le volume du stator pour l'autre.
\end{abstract}

\begin{abstract}
One uses a double space (with $z$ and $\theta$ ) associated with a temporal Fourier analysis of the magnetic fields and currents distribution occurring in an ironfree asynchronous cylindrical machine, assuming that the length of the rotor is infinite and that the stator has its real geometry. The analytical expressions of the fields are very easy to compute and they permit to calculate the characteristics.

One gives two expressions that permit the calculus of the active power by integration of the fields : at the surface of the rotor for one, in the volume of the stator for the other.
\end{abstract}

Le calcul des performances des machines électriques est un problème numérique difficile car il nécessite la résolution d'un problème tridimensionnel dépendant du temps. C'est pourquoi, il a été abordé par des modèles simplifiés. Pour des systèmes tridimensionnels, la méthode des éléments finis est maintenant bien connue dans le cas stationnaire $[1,2]$ et elle commence à s'appliquer en dynamique [3, 4]. En tridimensionnel, cette méthode se heurte en particulier à la lourdeur des calculs; des méthodes intégrales seront peut-être mieux adaptées.

Une technique assez différente consiste à effectuer la décomposition en séries de Fourier spatio-temporelles des diverses grandeurs du système (courants, champs magnétiques et électriques, ...). Appliquée à un modèle plan d'une machine sans fer [5], cette méthode s'est révélée particulièrement efficace (il faut noter qu'elle utilise directement les champs, sous la forme d'une seule variable complexe combinant les deux composantes, et qu'elle ne passe pas par l'intermédiaire des potentiels). Nous proposons ici d'étendre cette méthode pour permettre une première approche tridimensionnelle : alors que les études précédentes supposaient des machines infiniment longues, nous tenons compte des effets liés à la longueur finie du

(*) Equipe de Recherche Associée au CNRS no 838. stator (mais pas encore à celle du rotor) et calculons les performances à l'aide de décompositions spatiales en $z$ et $\theta$ et temporelle. Cet article expose le principe de la méthode, établit les équations de base et l'applique au cas particulier du calcul d'un moteur asynchrone sans fer alimenté par une source de courant sinusoïdale.

1. Problème. - Nous cherchons à calculer les performances (couple, puissance mécanique, puissance active, facteur de puissance, pertes, ...) d'une machine asynchrone ne comportant pas de matériau ferromagnétique dont le schéma est donné figure 1. Elle comprend :

- un rotor massif de rayon $R$ de conductivité $\sigma$, tournant à la vitesse angulaire $\Omega$,

- un bobinage polyphasé situé entre les rayons $S_{\mathrm{i}}$ et $S_{\mathrm{e}}$ dans lequel circulent des courants connus,

- une enceinte extérieure, matérielle ou non, de rayon intérieur $F$ (éventuellement très grand, auquel cas l'enceinte peut ne pas exister) sur laquelle sont données des conditions aux limites.

Les longueurs des diverses parties seront précisées plus loin.

Toutes les caractéristiques seront faciles à obtenir si l'on détermine le champ magnétique $\mathfrak{H}$ qui existe en tout point du système. Il nous suffit donc de 


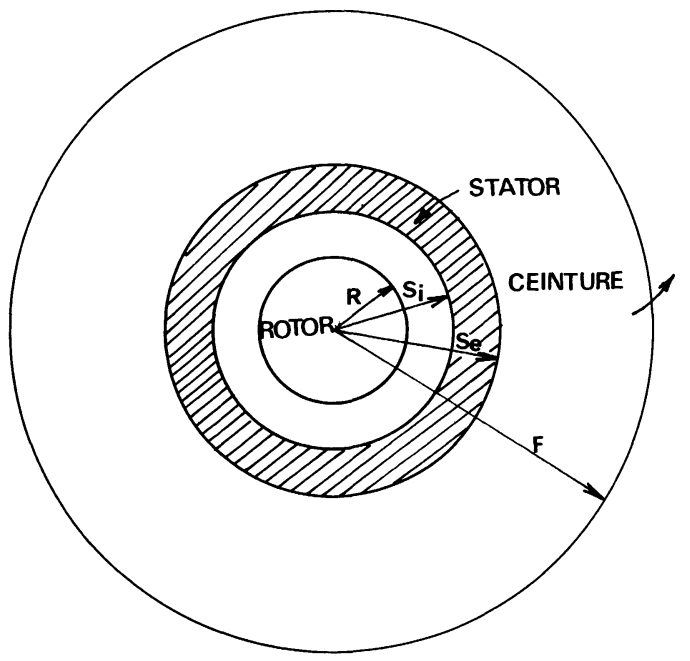

Fig. 1. - Section de la machine.

[The cross-section of the machine.]

résoudre les équations de Maxwell (dans le cadre de l'approximation électrotechnique) :

$$
\begin{aligned}
\nabla \wedge \mathfrak{H} & =\mathfrak{J} \\
\nabla . \mathfrak{H} & =0 \\
\nabla \wedge \boldsymbol{E} & =-\mu_{0} \frac{\partial \mathfrak{H}}{\partial t},
\end{aligned}
$$

en tenant compte des conditions aux limites.

La densité de courant $\mathfrak{J}$ est imposée dans le stator. Dans le rotor, elle provient de courants induits et obéit donc à la loi d'Ohm :

$$
\boldsymbol{J}=\sigma\left(\boldsymbol{E}+\mu_{0} \mathbf{v} \wedge \mathfrak{H}\right),
$$

où la vitesse locale $\mathbf{v}$ n'a qu'une composante $v_{\theta}=\Omega \rho$. Partout, compte tenu de (1a) :

$$
\nabla . \boldsymbol{J}=\mathbf{0} \text {. }
$$

2. Modélisation. - Le modèle étudié est représenté figure 2. Le rotor et l'enceinte sont infiniment longs. Des stators de longueur $H$ sont placés, de façon régulière, le long du rotor. Deux bobinages polyphasés consécutifs sont symétriques l'un de l'autre comme dans un miroir. Plus précisément, les bobinages 2 et 3 par exemple (cf. Fig. 2) sont symétriques par rapport au plan $z=0$ perpendiculaire à l'axe et l'on a :

$$
\begin{aligned}
& J_{r}(z, t)=J_{r}(-z, t) \\
& J_{\theta}(z, t)=J_{\theta}(-z, t) \\
& J_{z}(z, t)=-J_{z}(-z, t)
\end{aligned}
$$

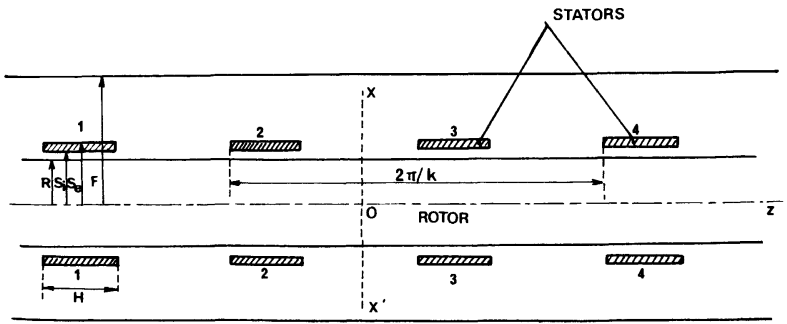

Fig. 2. - Les courants dans les stators 2 et 3 sont symétriques par rapport au plan $X^{\prime}$ et se retrouvent identiques dans les stators 2 et 4 (de même que dans 1 et 3 ).

[The currents in the stators 2 and 3 are symmetrical with respect to the plane $X^{\prime}$ and are identical in the stators 2 and 4 (and also in 1 and 3).]

$J(z, t)(z>0)$ représentant le courant dans le bobinage $3, \mathfrak{J}(-z, t)$ dans le bobinage 2 . Cette symétrie amène des simplifications dans la décomposition en série de Fourier en $z$ de J. La distance séparant deux bobinages est choisie suffisamment grande pour que leurs effets soient indépendants. Le pas du système comporte donc deux machines qui ajoutent leurs efforts sur le rotor : ce pas vaut $2 \pi / k$ où $k$ est le nombre d'onde. Ainsi, la machine réelle que l'on modélise correspond à un demi-pas. Le courant circulant dans les bobinages est périodique, de pulsation fondamentale $\omega$.

Dans le référentiel $r, \theta, z$, le système décrit se prête bien à une décomposition en série de Fourier complexe par rapport à $z, \theta$ et $t$. Comme dans l'étude bidimensionnelle [5], nous associerons, pour simplifier, les deux composantes radiale et azimuthale $\mho_{\rho}$ et $\vartheta_{\theta}$ de tout vecteur $\boldsymbol{v}$ de manière à former une seule grandeur complexe :

$$
v_{2}=v_{\rho}-i v_{\theta}
$$

On appellera $\vartheta_{3}=\vartheta_{\rho}+i \vartheta_{\theta}=\vartheta_{2}^{*}$.

Ceci donne :

$$
\vartheta_{\rho}=\frac{1}{2}\left(\vartheta_{2}+\vartheta_{3}\right) \quad v_{\theta}=\frac{i}{2}\left(\vartheta_{2}-\vartheta_{3}\right)
$$

Nous poserons de plus :

$$
v_{1}=v_{z}
$$

(4) Alors, nous écrirons la décomposition d'une grandeur ข $\left({ }^{1}\right)$ :

$$
\mathcal{V}(\rho, \theta, z, t)=\sum_{n, l, q=-\infty}^{+\infty} \mho_{n l q}=\sum V_{n l q}(\rho) \mathrm{e}^{i(n \omega t+l \theta+q k z)},
$$

avec

$$
V_{n l q}(\rho)=\frac{k \omega}{(2 \pi)^{3}} \int_{0}^{2 \pi / \omega} \int_{0}^{2 \pi} \int_{0}^{2 \pi / k} \vartheta(\rho, \theta, z, t) \mathrm{e}^{-i(n \omega t+l \theta+q k z)} \mathrm{d} t \mathrm{~d} \theta \mathrm{d} z
$$

$\left({ }^{1}\right)$ La notation $\mho$ (en lettre ronde) sera réservée aux grandeurs effectives et la notation $V$ (en lettre d'imprimerie) à leurs coefficients de Fourier. 
Remarquons que le calcul direct des coefficients relatifs à $\vartheta_{2}$ fournit, pour chaque ensemble $n l q$, une valeur complexe $V_{2 n l q}$ alors que le passage séparé par $\vartheta_{\rho}$ et $\vartheta_{\theta}$ donneraient deux complexes $V_{\rho n l q}$ et $V_{\theta n l q}$ soit deux fois plus de variables : nous voyons donc tout l'intérêt de l'introduction de $v_{2}$.

Pour toute variable réelle telle que $v_{1}$, on a $\vartheta_{1}=\mho_{1}^{*}$, soit en appliquant la décomposition (6) :

$$
V_{1,-n-l-q}^{*}=V_{1, n l q} \text {. }
$$

Par ailleurs :

$$
V_{3,-n-l-q}^{*}=V_{2, n l q}
$$

3. Les équations de base. - Dans les équations (1), on peut éliminer $\mathcal{E}$ à l'aide de la loi d'Ohm (2), puis remplacer $\mathfrak{H}$ et $\mathfrak{J}$ par leurs décompositions en série de Fourier. Si on multiplie alors chaque équation par $\exp [-i(n \omega t+l \theta+q k z)], n, p$ et $q$ étant fixés et si l'on intègre sur $t, \theta$ et $z$, il apparaît que les équations relatives aux diverses grandeurs $H_{1,2,3 n l q}$ et $I_{1,2,3 n l q}$ ne se couplent qu'entre elles. On obtient une formulation qui découple (apparemment seulement vu (9) et (10)) les modes $n l q$ et $-n,-l,-q$. On peut done écrire séparément l'évolution de chacun des modes et, pour simplifier la notation, le mode $n l q$ étant fixé, oublier dans l'écriture les indices $n l q$ : désormais, $V$ sera le coefficient de la série de Fourier de la grandeur $v$ pour des valeurs de nlq fixées.

Avec ces notations, les équations relatives à un mode $n l q$ donné s'écrivent très facilement à partir des équations écrites pour les grandeurs non encore décomposées en série de Fourier. Il suffit d'effectuèr la correspondance :

$$
\begin{gathered}
\frac{\partial}{\partial \theta} \rightarrow i l \quad \frac{\partial}{\partial z} \rightarrow i q k \quad \frac{\partial}{\partial t} \rightarrow \text { in } \omega \\
\vartheta_{1} \rightarrow V_{1}, \quad V_{\rho} \rightarrow \frac{1}{2}\left(V_{2}+V_{3}\right), \quad v_{\theta} \rightarrow \frac{i}{2}\left(V_{2}-V_{3}\right) .
\end{gathered}
$$

Il faut remarquer que les équations pour $\mathrm{H}_{3}$ et $I_{3}$, compte tenu de (10), s'obtiennent très simplement par transformation à partir de celles relatives à $\mathrm{H}_{2}$ et $\mathrm{I}_{2}$ : on change $n l q$ en $-n-l-q, i$ en $-i$ (pour la conjugaison), $H_{2}$ et $I_{2}$ en $H_{3}$ et $I_{3}, H_{1}$ et $I_{1}$ restant les mêmes (cf. (9)). Nous n'écrirons donc que les équations essentielles relatives à $H_{3}$ et $I_{3}$.

Ainsi, en effectuant la combinaison $-i(1 a)_{\rho}-(1 a)_{\theta}$, on obtient :

$$
\frac{\partial H_{1}}{\partial \rho}+\frac{l}{\rho} H_{1}-i q k H_{2}=-i I_{2}
$$

De même, $i(1 a)_{z}-(1 b)$ donne :

$$
-\frac{\partial H_{2}}{\partial \rho}+\frac{l-1}{\rho} H_{2}-i q k H_{1}=i I_{1}
$$

et, par simple transformation de (12) :

$$
-\frac{\partial H_{3}}{\partial \rho}-\frac{l+1}{\rho} H_{3}-i q k H_{1}=-i I_{1} .
$$

Notons que si l'on transforme (11) comme (12) l'a été pour donner (13), on obtient :

$$
\frac{\partial H_{1}}{\partial \rho}-\frac{l}{\rho} H_{1}-i q k H_{3}=i I_{3} .
$$

Soustrayant (11a) de (11) on obtient une relation intéressante, ne contenant plus de dérivée par rapport à $\rho$ :

$$
\frac{2 l}{\rho} H_{1}-i q k\left(H_{2}-H_{3}\right)=-i\left(I_{2}+I_{3}\right)
$$

qui traduit $(\nabla \wedge \mathfrak{X})_{\rho}=\mathfrak{J}_{\rho}$.

Les trois équations (11), (12) et (13) sont celles de base pour le mode $n l q$, mais elles donnent aussi les champs du mode $-n-l-q$ compte tenu de (9) et (10). Elles permettent de calculer les champs hors du rotor puisque $I_{1}$ et $I_{2}$ sont alors connus. Dans le rotor même, il faut leur associer les équations déterminant le courant qui est de type induit. On déduit de (1c) et (2) :

$$
\frac{1}{\sigma}(\nabla \wedge \mathfrak{J})=-\mu_{0} \frac{\partial \mathscr{X}}{\partial t}+\mu_{0} \nabla \wedge(\mathbf{A} \boldsymbol{X})
$$

car $\sigma$ étant constant dans le rotor,

$$
\nabla \wedge\left(\frac{\mathfrak{J}}{\sigma}\right)=\frac{1}{\sigma}(\nabla \wedge \mathfrak{\jmath}) .
$$

Calculant $\sigma\left[(15)-i(15)_{\theta}\right]$ et tenant compte de (1b), il vient :

$$
q k I_{2}+i \frac{l}{\rho} I_{1}+i \frac{\partial I_{1}}{\partial \rho}=-i \mu_{0} \sigma(n \omega+l \Omega) H_{2} .
$$

De même $\sigma(15)_{z}-i(3)$ donne :

$$
i \frac{l-1}{\rho} I_{2}+q k I_{1}-i \frac{\partial I_{2}}{\partial \rho}=i \sigma \mu_{0}(n \omega+l \Omega) H_{1} .
$$

4. Méthode de résolution - Le calcul des champs nécessite la résolution des équations linéaires couplées : (11), (12) et (13) seules hors rotor, associées à (1b) et (17) dans le rotor. Les conditions aux limites sont imposées et, puisque l'on traite séparément les domaines intérieur et extérieur au rotor (ce qui permet de ne pas introduire dans les équations la discontinuité en $\sigma$ à la surface du rotor), il faut raccorder les solutions au niveau de cette surface : nous y écrirons la continuité de $H_{1}, H_{2}$ et $H_{3}$. Ceci, par (14), entraîne celle de $I_{\rho}$. Comme juste à l'extérieur, on est dans 
l'entrefer : $I_{\rho}=0$ et, par suite, juste à l'intérieur à la surface du rotor, $I_{\rho}$ est nul : le courant est bien tangent à la surface du conducteur comme cela doit être.
Pour découpler les équations décrivant les évolutions de $H_{1}$ et $H_{2}$, il suffit de remplacer (11) et (12) par les équations du deuxième ordre qui s'en déduisent :

$$
\begin{aligned}
& \frac{\partial^{2} H_{1}}{\partial \rho^{2}}+\frac{1}{\rho} \frac{\partial H_{1}}{\partial \rho}-\left(\frac{l^{2}}{\rho^{2}}+q^{2} k^{2}\right) H_{1}=q k I_{1}+i \frac{l-1}{\rho} I_{2}-i \frac{\partial I_{2}}{\partial \rho}, \\
& \frac{\partial^{2} H_{2}}{\partial \rho^{2}}+\frac{1}{\rho} \frac{\partial H_{2}}{\partial \rho}-\left[\frac{(l-1)^{2}}{\rho^{2}}+q^{2} k^{2}\right] H_{2}=-i \frac{l}{\rho} I_{1}-q k I_{2}-i \frac{\partial I_{1}}{\partial \rho} .
\end{aligned}
$$

Ces équations décrivent le système hors rotor. Dans le rotor il faut leur associer (17) et (16) ce qui donne immédiatement pour $H_{1}$ :

$$
\frac{\partial^{2} H_{1}}{\partial \rho^{2}}+\frac{1}{\rho} \frac{\partial H_{1}}{\partial \rho}-H_{1}\left[\frac{l^{2}}{\rho^{2}}+q^{2} k^{2}+i \sigma \mu_{0}(n \omega+l \Omega)\right]=0 .
$$

Pour $H_{2}$, on tient compte en plus de (12) et de (13) et l'on obtient :

$$
\frac{\partial^{2} H_{2}}{\partial \rho^{2}}+\frac{1}{\rho} \frac{\partial H_{2}}{\partial \rho}-\left[\frac{(l-1)^{2}}{\rho^{2}}+q^{2} k^{2}+i \sigma \mu_{0}(n \omega+l \Omega)\right] H_{2}=0,
$$

et, par transformation, pour $\mathrm{H}_{3}$ :

$$
\frac{\partial^{2} H_{3}}{\partial \rho^{2}}+\frac{1}{\rho} \frac{\partial H_{3}}{\partial \rho}-\left[\frac{(l+1)^{2}}{\rho^{2}}+q^{2} k^{2}+i \sigma \mu_{0}(n \omega+l \Omega)\right] H_{3}=0 .
$$

Le remplacement de trois équations du premier ordre par trois du deuxième ordre fait perdre de l'information. Il faudra assurer que les solutions obtenues satisfont toujours bien les relations du premier ordre.

Nous allons maintenant effectuer quelques changements de variables et de notations. Nous poserons :

- hors rotor

$$
\begin{aligned}
Z & =i q k \rho=a \rho, \\
H_{1,2,3}^{\prime} & =\frac{\partial H_{1,2,3}}{\partial Z} \\
\text { on a donc } Z H_{1,2,3}^{\prime} & \left.=\rho \frac{\partial H_{1,2,3}}{\partial \rho}\right), \\
I_{1}^{\prime} & =\frac{\partial I_{1}}{\partial z} \quad I_{2}^{\prime}=\frac{\partial I_{2}}{\partial z}, \\
T_{1} & =\frac{1}{q k}\left(-I_{1}+\frac{l-1}{Z} I_{2}-I_{2}^{\prime}\right), \\
T_{2} & =\frac{1}{q k}\left(-\frac{l}{Z} I_{1}+I_{2}-I_{1}^{\prime}\right), \\
T_{3} & =\frac{1}{q k}\left(-\frac{l}{Z} I_{1}-I_{3}+I_{1}^{\prime}\right),
\end{aligned}
$$

- dans le rotor

$$
\begin{gathered}
Z_{R}^{2}=-\left[q^{2} k^{2}+i \sigma \mu_{0}(n \omega+l \Omega)\right] \rho^{2}=b^{2} \rho^{2}, \\
H_{1,2,3}^{\prime}=\frac{\partial H_{1,2,3}}{\partial Z_{R}} \quad\left(\operatorname{avec} Z_{R} H_{1,2,3}^{\prime}=\rho \frac{\partial H_{1,2,3}}{\partial \rho}\right) .
\end{gathered}
$$


Les équations s'écrivent ainsi :

- hors rotor

$$
\begin{aligned}
H_{1}^{\prime \prime}+\frac{1}{Z} H_{1}^{\prime}+\left(1-\frac{l^{2}}{Z^{2}}\right) H_{1} & =T_{1}, \\
H_{2}^{\prime \prime}+\frac{1}{Z} H_{2}^{\prime}+\left[1-\frac{(l-1)^{2}}{Z^{2}}\right] H_{2} & =T_{2}, \\
H_{3}^{\prime \prime}+\frac{1}{Z} H_{3}^{\prime}+\left[1-\frac{(l+1)^{2}}{Z^{2}}\right] H_{3} & =T_{3},
\end{aligned}
$$

— dans le rotor

$$
\begin{aligned}
H_{1}^{\prime \prime}+\frac{1}{Z_{R}} H_{1}^{\prime}+\left(1-\frac{l^{2}}{Z_{R}^{2}}\right) & =0, \\
H_{2}^{\prime \prime}+\frac{1}{Z_{R}} H_{2}^{\prime}+\left(1-\frac{(l-1)^{2}}{Z_{R}^{2}}\right) & =0, \\
H_{3}^{\prime \prime}+\frac{1}{Z_{R}} H_{3}^{\prime}+\left(1-\frac{(l+1)^{2}}{Z_{R}^{2}}\right) & =0 .
\end{aligned}
$$

On a donc affaire à des équations de Bessel comportant éventuellement un second membre.

5. Expressions générales des champs. - Dans le rotor, les solutions générales sont des combinaisons linéaires des fonctions de Bessel $J$ et $Y$ :

$$
\begin{aligned}
& H_{1}=A_{1} J_{l}(b \rho)+\tilde{A}_{1} Y_{l}(b \rho), \\
& H_{2}=A_{2} J_{l-1}(b \rho)+\tilde{A}_{2} Y_{l-1}(b \rho), \\
& H_{3}=A_{3} J_{l+1}(b \rho)+\tilde{A}_{3} Y_{l+1}(b \rho) .
\end{aligned}
$$

Hors rotor, les équations comportent un second membre. Les solutions peuvent s'obtenir en appliquant la méthode de la variation de la constante (7). Nous allons expliciter le calcul pour $H_{1}$. On pose :

$$
H_{1}=\lambda_{1}(Z) J_{l}(Z)+\mu_{1}(Z) Y_{l}(Z) .
$$

On remplace ainsi la fonction inconnue $H_{1}$ par un ensemble de deux fonctions $\lambda_{1}(Z)$ et $\mu_{1}(Z)$ liées par une relation supplémentaire pratique :

$$
\lambda_{1}^{\prime} J_{l}+\mu_{1}^{\prime} Y_{l}=0
$$

On peut alors écrire simplement :

$$
\begin{aligned}
& H_{1}^{\prime}=\lambda_{1} J_{l}^{\prime}+\mu_{1} Y_{l}^{\prime}, \\
& H_{1}^{\prime \prime}=\lambda_{1} J_{l}^{\prime \prime}+\mu_{1} Y_{l}^{\prime \prime}+\lambda_{1}^{\prime} J_{l}^{\prime}+\mu_{1}^{\prime} Y_{l}^{\prime} .
\end{aligned}
$$

Reportant dans (30), il reste :

$$
\lambda_{1}^{\prime} J_{l}^{\prime}+\mu_{1}^{\prime} Y_{l}^{\prime}=T_{1}
$$

$\lambda_{1}^{\prime}$ et $\mu_{1}^{\prime}$ sont donc déterminés par (39) et (40). Le wronskien ayant une valeur simple (6) :

$$
J_{l} Y_{l}^{\prime}-Y_{l} J_{l}^{\prime}=2 / \pi Z
$$

on en déduit :

$$
\begin{aligned}
& \lambda_{1}^{\prime}=-\frac{\pi}{2} Z Y_{l} T_{1}, \\
& \mu_{1}^{\prime}=\frac{\pi}{2} Z J_{l} T_{1} .
\end{aligned}
$$


$T_{1}$ est donné par l'expression (25). Les courants n'existant que dans le stator, $T_{1}$ est nul en dehors et l'on a donc :

$$
\lambda_{1}=G_{1}-\frac{\pi}{2} \int_{a R}^{a \rho} Z Y_{l} T_{1} \mathrm{~d} Z=G_{1}-\frac{\pi}{2 q k} \int_{a R}^{a \rho} Z Y_{l}\left(-I_{1}+\frac{l-1}{z} I_{2}-I_{2}^{\prime}\right) \mathrm{d} Z,
$$

où $G_{1}$ est une constante.

Pour évaluer l'intégrale, remarquons que :

$$
\left(Z Y_{l} I_{2}\right)^{\prime}=Y_{l} I_{2}+Z Y_{l}^{\prime} I_{2}+Z Y_{l} I_{2}^{\prime},
$$

et que, d'après les propriétés des fonctions de Bessel [6] :

$$
Y_{l}^{\prime}(Z)=Y_{l-1}(Z)-\frac{l}{Z} Y_{l}(Z)
$$

Il vient successivement :

$$
\begin{aligned}
\int_{a R}^{a \rho} Z Y_{l} T_{1} \mathrm{~d} Z & =\frac{1}{q k} \int_{a R}^{a \rho}\left[-Z Y_{l} I_{1}+l I_{2} Y_{l}+Z Y_{l}^{\prime} I_{2}-\left(Z Y_{l} I_{2}\right)^{\prime}\right] \mathrm{d} Z \\
& =\frac{1}{q k}\left\{-\int_{a R}^{a \rho}\left(I_{1} Y_{l}-I_{2} Y_{l-1}\right) Z \mathrm{~d} Z-a \rho Y_{l} I_{2}(\rho)\right\}
\end{aligned}
$$

$\left(\operatorname{car} I_{2}(R)=0\right)$.

On calcule $\mu_{1}$ de la même façon et l'on obtient l'expression de $H_{1}$. Le même type de calcul donne $H_{2}$ et $H_{3}$. Dans tous ces calculs, il apparait des intégrales (les mêmes pour $H_{2}$ et $H_{1}$ ) et nous poserons :

$$
\begin{aligned}
& C_{1}(\rho)=\frac{\pi}{2 q k} \int_{a R}^{a \rho}\left(I_{1} Y_{l}-I_{2} Y_{l-1}\right) Z \mathrm{~d} Z, \\
& \tilde{C}_{1}(\rho)=-\frac{\pi}{2 q k} \int_{a R}^{a \rho}\left(I_{1} J_{l}-I_{2} J_{l-1}\right) Z \mathrm{~d} Z, \\
& C_{3}(\rho)=\frac{\pi}{2 q k} \int_{a R}^{a \rho}\left(I_{1} Y_{l}+I_{3} Y_{l+1}\right) Z \mathrm{~d} Z, \\
& \tilde{C}_{3}(\rho)=-\frac{\pi}{2 q k} \int_{a R}^{a \rho}\left(I_{1} J_{l}+I_{3} J_{l+1}\right) Z \mathrm{~d} Z,
\end{aligned}
$$

avec, après quelques calculs utilisant $\mathbf{\nabla . I}=0$

$$
\begin{aligned}
& C_{1}(\rho)-C_{3}(\rho)=-\frac{i \pi}{2} \rho Y_{l}(a \rho)\left(I_{2}(\rho)+I_{3}(\rho)\right), \\
& \tilde{C}_{1}(\rho)-\tilde{C}_{3}(\rho)=-\left(C_{1}(\rho)-C_{3}(\rho)\right) .
\end{aligned}
$$

Notons que : si $C$ représente $C_{1}, \widetilde{C}_{1}, C_{3}$ ou $\tilde{C}_{3}$

$$
\begin{aligned}
& C\left(\rho<S_{\mathrm{i}}\right)=0 \\
& C\left(\rho>S_{\mathrm{e}}\right)=C\left(Z_{\mathrm{e}}\right)
\end{aligned}
$$

et

$$
I_{2}\left(\rho<S_{\mathrm{i}}\right)=0 .
$$

Ainsi, nous avons :

$$
\begin{aligned}
& H_{1}(a \rho)=\left(G_{1}+C_{1}(\rho)\right) J_{l}(a \rho)+\left(\tilde{G}_{1}+\tilde{C}_{1}(\rho)\right) Y_{l}(a \rho), \\
& H_{2}(a \rho)=\left(G_{2}+C_{1}(\rho)\right) J_{l-1}(a \rho)+\left(\tilde{G}_{2}+\tilde{C}_{1}(\rho)\right) Y_{l-1}(a \rho), \\
& H_{3}(a \rho)=\left(G_{3}+C_{3}(\rho)\right) J_{l+1}(a \rho)+\left(\tilde{G}_{3}+\tilde{C}_{3}(\rho)\right) Y_{l+1}(a \rho) .
\end{aligned}
$$


6. Détermination des constantes des champs. - Les douze constantes $A_{1}, \ldots, G_{1}, \ldots$ apparaissant dans les expressions (36), (37), (38), (47), (48), (49) seront déterminées par les conditions aux limites et les relations (11), (12), (13) comportant les dérivées du premier ordre.

Les conditions aux limites sont les suivantes:

- Au centre, le champ doit être fini. Or $Y_{l}(z) \rightarrow \infty$ pour $z \rightarrow 0$; on doit donc avoir

$$
\tilde{A}_{1}=\tilde{A}_{2}=\tilde{A}_{3}=0 \text {. }
$$

- A la surface du rotor, les champs peuvent être considérés comme étant « hors rotor " ou " dans le rotor " mais ils doivent avoir la même valeur, ce qui donne,

$$
\begin{aligned}
A_{1} J_{l}(b R) & =G_{1} J_{l}(a R)+\tilde{G}_{1} Y_{l}(a R), \\
A_{2} J_{l-1}(b R) & =G_{2} J_{l-1}(a R)+\tilde{G}_{2} Y_{l-1}(a R), \\
A_{3} J_{l+1}(b R) & =G_{3} J_{l+1}(a R)+\tilde{G}_{3} Y_{l+1}(a R) .
\end{aligned}
$$

- A la surface limite de rayon $F$, le champ est imposé par la nature et/ou les dimensions de l'enceinte.

S'il n'y a pas d'enceinte, c'est-à-dire si $F \rightarrow \infty$, le champ doit être nul pour $\rho=F \rightarrow \infty$, ce qui impose que les solutions soient du type $J_{l}+i Y_{l}$ (fonction de Hankel $H_{l}^{(1)}$ ) et conduit à :

$$
\begin{aligned}
& G_{1}+C_{1}\left(S_{\mathrm{e}}\right)=-i\left(\tilde{G}_{1}+\tilde{C}_{1}\left(S_{\mathrm{e}}\right)\right), \\
& G_{2}+C_{1}\left(S_{\mathrm{e}}\right)=-i\left(G_{2}+C_{1}\left(S_{\mathrm{e}}\right)\right), \\
& G_{3}+C_{3}\left(S_{\mathrm{e}}\right)=-i\left(G_{3}+C_{3}\left(S_{\mathrm{e}}\right)\right) .
\end{aligned}
$$

Si $F$ est comparable à $S_{\mathrm{e}}$, il est essentiel de connaitre la nature de l'enceinte. Nous étudierons le cas où elle est formée de matériau ferromagnétique à très forte perméabilité; le champ magnétique est donc normal à la surface; ses composantes tangentielles sont nulles :

$$
\begin{aligned}
H_{1}(a F) & =0, \\
H_{2}(a F)-H_{3}(a F) & =0 .
\end{aligned}
$$

Considérons maintenant les relations du premier ordre. Ecrivons (11), (12), (13) dans l'entrefer, pour $R \leqslant \rho \leqslant S_{\mathrm{i}}$, là où les courants et les intégrales $C(\rho)$ sont nuls. Il vient, tout d'abord à partir de (11) :

$$
G_{1} J_{l}^{\prime}+\tilde{G}_{1} Y_{l}^{\prime}+\frac{l}{Z} G_{1} J_{l}+\frac{l}{Z} \tilde{G}_{1} Y_{l}-G_{2} J_{l-1}-\tilde{G}_{2} Y_{l-1}=0,
$$

soit, avec (41) :

ou encore

$$
\left(G_{1}-G_{2}\right) J_{l-1}+\left(\tilde{G}_{1}-\widetilde{G}_{2}\right) Y_{l-1}=0
$$

$$
\begin{aligned}
& G_{1}=G_{2} \\
& \tilde{G}_{1}=\tilde{G}_{2},
\end{aligned}
$$

(12) redonne la même condition et (13) donne :

$$
\begin{aligned}
& G_{1}=-G_{3}, \\
& \tilde{G}_{1}=-\tilde{G}_{3} .
\end{aligned}
$$

Il est facile de vérifier que si (55) et (56) sont vérifiées, les relations du premier ordre sont complètement satisfaites hors du rotor, y compris pour $\rho \geqslant S_{\text {i }}$.

Considérons de près les conditions (53), (55) et (56) valables pour une machine sans ceinture. On voit que (53a) associée à (55) donne (53b) et (56) associée à (53a) donne (53c) lorsque l'on tient compte de (47). Les relations $(53 b)$ et $(53 c)$ sont redondantes avec les précédentes et ne doivent pas être conservées en tant que condition à respecter.

De même, pour une machine avec une enceinte on a (54), (55) et (56). Les deux dernières entraînent que les relations du premier ordre, et en particulier (14) sont valables. Si on écrit (14) pour $\rho=F$, rayon pour lequel $I_{2}=I_{3}=0$, on a simplement :

$$
\frac{2 l}{\rho} H_{1}-i q k\left(H_{2}-H_{3}\right)=0 .
$$

qui, avec (54a), redonne (54b). (54b) n'est donc pas une condition à conserver. 
Dans le rotor, deux des trois relations (11), (12), (13) vont servir à calculer (si on le désire) les courants induits $I_{1}$ et $I_{2}$, la troisième (éventuellement combinée avec les autres) restant comme une condition à vérifier. Nous prendrons en pratique comme condition la relation obtenue en additionnant (12) et (13) car elle ne fait plus intervenir les courants; elle s'écrit :

$$
Z_{R}\left(A_{2} J_{l-1}^{\prime}+A_{3} J_{l+1}^{\prime}\right)-(l-1) A_{2} J_{l-1}+(l+1) A_{3} J_{l+1}+2 Z A_{1} J_{l}=0 .
$$

Or les dérivées des fonctions de Bessel obéissent à [6] :

$$
\begin{aligned}
& J_{l+1}^{\prime}\left(Z_{R}\right)=J_{l}\left(Z_{R}\right)-\frac{l+1}{Z_{R}} J_{l+1}\left(Z_{R}\right), \\
& J_{l-1}^{\prime}\left(Z_{R}\right)=-J_{l}\left(Z_{R}\right)+\frac{l-1}{Z_{R}} J_{l-1}\left(Z_{R}\right) .
\end{aligned}
$$

(57) devient ainsi :

$$
-Z_{R} A_{2} J_{l}+Z_{R} A_{3} J_{l}+2 Z A_{1} J_{l}=0,
$$

ce qui, avec $Z / Z_{R}=a / b$ donne la relation suivante entre les coefficients :

$$
2 a A_{1}-b A_{2}+b A_{3}=0 .
$$

7. Expression finale des composantes de Fourier des champs. - Les champs valent :

- dans le rotor

$$
\begin{aligned}
& H_{1}=A_{1} J_{l}(b \rho) \\
& H_{2}=A_{2} J_{l-1}(b \rho) \\
& H_{3}=A_{3} J_{l+1}(b \rho),
\end{aligned}
$$

- hors rotor

$$
\begin{aligned}
& H_{1}=\left(G_{1}+C_{1}\right) J_{l}(a \rho)+\left(\tilde{G}_{1}+\tilde{C}_{1}\right) Y_{l}(a \rho), \\
& H_{2}=\left(G_{1}+C_{1}\right) J_{l-1}(a \rho)+\left(\tilde{G}_{1}+\tilde{C}_{1}\right) Y_{l-1}(a \rho), \\
& H_{3}=\left(-G_{1}+C_{3}\right) J_{l+1}(a \rho)+\left(-\tilde{G}_{1}+\tilde{C}_{1}\right) Y_{l+1}(a \rho) .
\end{aligned}
$$

Ils dépendent de cinq constantes telles que :

$$
\begin{aligned}
2 a A_{1}-b A_{2}+b A_{3} & =0, \\
A_{1} J_{l}(b R)-G_{1} J_{l}(a R)-\tilde{G}_{1} Y_{l}(a R) & =0, \\
A_{2} J_{l-1}(b R)-G_{1} J_{l-1}(a R)-\tilde{G}_{1} Y_{l-1}(a R) & =0, \\
A_{3} J_{l+1}(b R)+G_{1} J_{l+1}(a R)+\tilde{G}_{1} Y_{l+1}(a R) & =0,
\end{aligned}
$$

et

- si la machine est entourée d'une ceinture magnétique de rayon $F$ :

$$
\left(G_{1}+C_{1}\left(S_{\mathrm{e}}\right)\right) J_{l}(a F)+\left(\widetilde{G}_{1}+\widetilde{C}_{1}\left(S_{\mathrm{e}}\right)\right) Y_{l}(a F)=0,
$$

que l'on écrira

$$
G_{1} J_{l}(a F)+\tilde{G}_{1} Y_{l}(a F)=-C_{1}\left(S_{\mathrm{e}}\right) J_{l}(a F)-\tilde{C}_{1}\left(S_{\mathrm{e}}\right) Y_{l}(a F),
$$

- si la machine est seule :

$$
G_{1}+i \widetilde{G}_{1}=C_{1}\left(S_{\mathrm{e}}\right)+i \widetilde{C}_{1}\left(S_{\mathrm{e}}\right) .
$$

Les cinq constantes s'obtiennent donc en résolvant un système de cinq équations linéaires, ce qui est facile numériquement. On obtiendra ainsi les champs magnétiques d'ordre $n, l, q$ créés par des courants statoriques dont on s'est donné les valeurs $I_{1}(\rho), I_{2}(\rho)$ et $I_{3}(\rho)$ pour le mode considéré et simultanément ceux d'ordre - $n$, $-l,-q$ compte tenu de (9) et (10). 
8. Description du bobinage. - Le bobinage statorique est en général composé de $p$ paires de pôles, chacune comportant $m$ phases, c'est-à-dire $m$ bobines alimentées par des courants déphasés convenablement dans le temps. Les bobines sont fabriquées avec un fil de conductivité $\sigma$, de section $s$, qui a pour direction $\mathbf{u}$ (u étant un vecteur unitaire) au point $r, \theta, z$ du stator, et qui est parcouru par un courant de densité $J(t)$.

Pour la première bobine de la première paire de pôles, on écrit :

$$
\mathfrak{J}_{1,1}(r, \theta, z, t)=\mathbf{u}(r, \theta, z) \mathfrak{J}(\omega t)
$$

la description géométrique de cette bobine étant entièrement donnée par la fonction $\mathbf{u}(r, \theta, z)$. (|u $\mid=1$ là où la première bobine existe, $\mathbf{u}=0$ ailleurs.)

La décomposition en série de Fourier de $\mathfrak{J}_{1,1}$ s'écrit :

$$
J_{1,1}=\sum_{n, l, q} I_{n} \mathbf{u}_{l q}(\rho) \mathrm{e}^{i(n \omega t+l \theta+q k z)}
$$

Toutes les bobines du stator sont identiques. Elles sont au nombre de $m p$ et se déduisent les unes des autres par simple décalage spatial angulaire. Pour la bobine $m_{i}$ de la paire de pôles $p_{j}$, alimentée avec un courant déphasé temporellement par rapport à celui de la précédente bobine, on a :

$$
\mathfrak{J}_{i}, p_{j}=\mathbf{u}\left(r, \theta-\frac{p_{j}-1}{p} 2 \pi-\frac{m_{i}-1}{m p} 2 \pi, z\right) I\left(\omega t-\frac{m_{i}-1}{m} 2 \pi\right),
$$

dont la décomposition s'écrit :

$$
J_{m_{i}}, p_{j}=\sum_{n l q} I_{n} \mathbf{u}_{l q}(\rho) \mathrm{e}^{i(n \omega t+l \theta+q k z)} \exp \left\{-2 i \pi\left[l \frac{p_{j}-1}{p}+\frac{m_{i}-1}{m}\left(\frac{l}{p}+n\right)\right]\right\} .
$$

Les champs créés par les divers bobinages s'ajoutent. Or, les composantes de Fourier $\mathscr{H}_{\text {nlq }}$ des champs créés par chacun des bobinages sont proportionnelles aux composantes de Fourier des courants les traversant : elles sont donc toutes proportionnelles à $I_{n} \mathbf{u}_{l q} \mathrm{e}^{i(n \omega t+l \theta+q k z)}$ et ne diffèrent que par un déphasage. Si on connaît $\mathcal{H}_{1 n l q}\left(\right.$ et $\left.\mathcal{H}_{2 n l q}\right)$ créés par la première bobine, on aura pour le champ total :

$$
\mathcal{H}_{1 n l q}^{t}=\mathcal{H}_{1 n l q} \sum_{m_{j}=1}^{m} \sum_{p_{i}=1}^{p} \exp -2 i \pi\left[l \frac{p_{j}-1}{p}+\left(\frac{l}{p}+n\right) \frac{m_{i}-1}{m}\right] .
$$

Pour que la double sommation ne soit pas nulle, il faut que :

$$
\begin{array}{ll}
l=r p & \text { où } r \text { est un entier }, \\
\frac{l}{p}+n=f m & \text { où } f \text { est un entier, }
\end{array}
$$

auquel cas :

et de même :

$$
\mathcal{H}_{1 n l q}^{t}=\mathscr{H}_{1 n l q} \cdot m p
$$

$$
\mathcal{H}_{2 n l q}^{t}=\mathcal{H}_{2 n l q} \cdot m p \text {. }
$$

Le calcul du champ global s'obtient donc à partir du calcul du champ créé par une bobine en ne conservant que les harmoniques vérifiant les relations (65) et en multipliant le résultat par $m p$ (nombre de phases $\times$ nombre de paires de pôles).

Remarquons que, dans cette description, un bobinage formé de $m p$ bobines symétriques par rapport à l'axe, ce qui est l'un des cas le plus habituel, peut être décrit de deux façons :

- on effectue la décomposition en série de Fourier d'une bobine complète (qui ne comportera qu'un harmonique en $\theta$ sur deux, vue la symétrie) et l'on considère que l'on a $p$ paires de pôles et $m$ phases ;

- on effectue la décomposition en série de Fourier d'une demi-bobine (et l'on aura tous les harmoniques en $\theta$ ) et l'on considère que l'on a $p$ paires de pôles et $2 m$ phases (ce qui supprimera des harmoniques dans le calcul final, par application des relations (65)). Les phases supplémentaires sont en fait les autres demi-bobines qui sont alimentées par les courants retour.

Cette deuxième description est la seule possible pour le système à une paire de pôles ayant deux phases en quadrature qui est donc représenté par $m=4, p=1$ à partir d'une demi-bobine. 
9. Calcul du couple. - Le couple mécanique agissant sur le rotor vaut :

$$
\mathcal{C}=\int_{S} \mathbf{R} \wedge \mathscr{F} R \mathrm{~d} \theta \mathrm{d} z
$$

où $\mathfrak{F}$ est la force agissant sur le rotor. L'intégrale est effectuée sur la surface du rotor : nous intégrerons en $z$ de 0 à $2 \pi / k$ (cf. Fig. 1), soit sur deux machines et diviserons le résultat par 2 . Si le bobinage d'une machine est symétrique par rapport à un plan perpendiculaire à l'axe et passant par le centre (ce qui est le cas général), $\mathfrak{C}$ est porté par $\mathrm{O} z$ et son module vaut :

$$
\mathcal{C}=\frac{R^{2}}{2} \int_{0}^{2 \pi / k} \mathrm{~d} z \int_{0}^{2 \pi} \mathrm{d} \theta \mathcal{F}_{\theta}
$$

avec

$$
\mathscr{F}_{\theta}=-\mu_{0} m^{2} p^{2} \mathscr{H}_{\rho} \mathscr{H}_{\theta}=-\frac{\mu_{0} m^{2} p^{2} i}{4}\left(\mathcal{H}_{1}+\mathcal{H}_{2}^{*}\right)\left(\mathcal{H}_{1}-\mathcal{H}_{2}^{*}\right)
$$

Si on développe les champs en série de Fourier, il vient, après intégration :

$$
\mathcal{C}=-\frac{i \pi^{2} R^{2}}{2 k} \mu_{0} m^{2} p^{2} \sum_{n l q}\left(H_{2 n l q}+H_{2-n-l-q}^{*}\right)\left(H_{2-n-l-q}-H_{2 n l q}^{*}\right)=\sum_{n l q} C_{n l q},
$$

$n, l, q$ variant de $-\infty$ à $+\infty$.

Remarquons que :

$$
\begin{aligned}
\left(H_{2 n l q}+H_{2-n-l-q}^{*}\right)\left(H_{2-n-l-q}-H_{2 n l q}^{*}\right)+ & \left(H_{2-n-l-q}+H_{2 n l q}^{*}\right)\left(H_{2 n l q}-H_{2-n-l-q}^{*}\right)= \\
& =2\left(H_{2 n l q} H_{2-n-l-q}-H_{2 n l q}^{*} H_{2-n-l-q}^{*}\right)=4 i J m\left(H_{2 n l q} H_{2-n-l-q}\right) .
\end{aligned}
$$

Il vient donc (comme $q$ ne prend pas la valeur 0 ), compte tenu de (10) :

$$
\mathcal{C}=\frac{2 \pi^{2} R^{2}}{k} \mu_{0} m^{2} p^{2} \sum_{q>0} \sum_{n, l=-\infty}^{+\infty} J m\left(H_{2} H_{3}^{*}\right)_{n l q}=\sum_{q>0} \sum_{n, l} C_{n l q}
$$

10. Puissances à partir du couple. - Pour un mode nlq donné, on a la relation suivante entre la puissance mécanique $P_{m, n t q}$, la puissance active $P_{a, n l q}$ (dont on a soustrait les pertes joules dans le stator) et les pertes joules dans le rotor $P_{J R, n l q}$ :

$$
\begin{gathered}
P_{J R, n l q}=P_{a, n l q}-P_{m, n l q}, \\
\frac{P_{m, n l q}}{l \Omega}=\frac{P_{a, n l q}}{n \omega}=\frac{P_{J R, n l q}}{n \omega-l \Omega},
\end{gathered}
$$

et de plus :

$$
P_{m, n l q}=C_{n l q} \Omega
$$

On en déduit :

$$
P_{a, n l q}=C_{n l q} \frac{n \omega}{l}
$$

et de même :

$$
P_{a,-n-l-q}=C_{-n-l-q} \frac{n \omega}{l} .
$$

Les diverses puissances valent donc :

$$
\begin{aligned}
\mathfrak{T}_{m} & =\mathrm{C} \Omega \\
\mathfrak{T}_{a} & =\sum_{q>0} \sum_{n, p} C_{n l q} \cdot \frac{n \omega}{l}, \\
\mathfrak{T}_{J R} & =\mathfrak{T}_{a}-\mathfrak{T}_{m} .
\end{aligned}
$$


11. Tension. - La tension générée aux bornes d'un bobinage vaut :

$$
\mathcal{U}=-\frac{\partial \phi}{\partial t}=-\frac{\partial}{\partial t} \int_{S} \mathbf{B} \cdot \mathrm{d} \mathbf{S}
$$

$S$ étant une surface quelconque s'appuyant sur le fil du bobinage. Pour un élément dl de fil d'extrémités $\mathrm{A}$ et $\mathrm{B}$, nous définirons la surface $\mathrm{d} S$ correspondante par $\mathrm{AB}$, les deux parallèles $\mathrm{AA}^{\prime}, \mathrm{BB}^{\prime}$ à l'axe oz issues de $\mathrm{A}$ et $\mathrm{B}$ jusqu'à la cote $z=2 \pi / k$ et les deux rayons $\mathrm{A}^{\prime} \mathrm{O}^{\prime}$ et $\mathrm{B}^{\prime} \mathrm{O}^{\prime}$ (cf. Fig. 3). La surface $S$ s'obtiendra par addition des divers $\mathrm{d} S$.

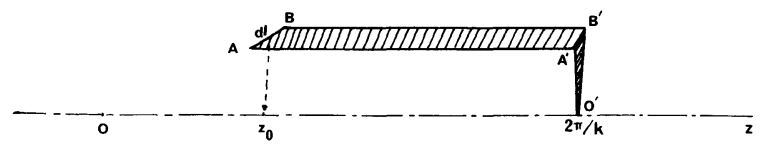

Fig. 3. - La surface $\mathrm{d} S$ de calcul du flux.

[The surface $\mathrm{d} S$ for the flux calculus.]

\section{Posons :} du fil.

$-\mathrm{d} \tau=s \mathrm{~d} l: \mathrm{d} \tau$ est le volume de l'élément de fil AB qui a pour section $s$, laquelle est constante tout au long

- $\mathbf{d l}=\mathbf{u} \mathrm{d} l: \mathbf{u}$ est un vecteur unitaire qui définit la direction du conducteur et par là même celle du courant.

Alors :

$$
\mathrm{d} u=-\frac{\partial}{\partial t} \int_{\mathrm{d} S} \mathbf{B} . \mathbf{d l} \wedge \mathbf{d z}=-\frac{\mathrm{d} \tau}{S} \int_{z_{0}}^{2 \pi / k}\left(\frac{\partial \mathbf{B}}{\partial t} \wedge \mathbf{u}\right) \cdot \mathbf{d z}
$$

Ainsi la tension élémentaire du est proportionnelle au volume élémentaire du conducteur. On aura la tension totale en intégrant sur le volume total des conducteurs, c'est-à-dire sur l'espace entier puisque $u$ n'est non nul que là où existent les conducteurs du bobinage :

$$
u=\int \mathrm{d} U=-\frac{1}{s} \int_{S_{\mathrm{i}}}^{S_{\mathbf{e}}} \rho \mathrm{d} \rho \int_{0}^{2 \pi} \mathrm{d} \theta \int_{0}^{2 \pi / k} \mathrm{~d} z_{0} \int_{z_{0}}^{2 \pi / k}\left(\frac{\partial \mathbf{B}}{\partial t} \wedge \mathbf{u}\right) \cdot \mathbf{d z} .
$$

En remarquant que :

$$
\left(\frac{\partial \mathbf{B}}{\partial t} \wedge \mathbf{u}\right) \cdot \mathbf{d z}=\left(\frac{\partial B_{\rho}}{\partial t} u_{\theta}-\frac{\partial B_{\theta}}{\partial t} u_{\rho}\right) \mathrm{d} z
$$

et en remplaçant $\mathbf{B}$ et $\mathbf{u}$ par leurs décompositions en série de Fourier, il vient, pour la première partie de l'intégrale :

$$
\int_{z_{0}}^{2 \pi / k} \frac{\partial B_{\rho}}{\partial t} u_{\theta} \mathrm{d} z=\frac{i}{4} \sum_{n l q l^{\prime} q^{\prime}} \int_{z_{0}}^{2 \pi / k}\left(B_{2}+B_{3}\right)_{n l q} \exp [i(n \omega t+l \theta+q k z)] \times\left(u_{2}-u_{3}\right)_{l^{\prime} q^{\prime}} \exp \left[i\left(l^{\prime} \theta+q^{\prime} k z\right)\right] \mathrm{d} z .
$$

La seconde partie s'explicite de la même façon.

Dans $(68 b)$, l'intégration en $z$ est alors immédiate, elle donne un terme en $\left(1-\exp i q k z_{0}\right)$. Celle en $z_{0}$ fait intervenir :

$$
\int_{0}^{2 \pi / k} \mathrm{~d} z_{0}\left(1-\mathrm{e}^{i q k z_{0}}\right) \mathrm{e}^{i q^{\prime} z_{0}}
$$

Comme $q^{\prime} \neq 0$, il faut que $q+q^{\prime}=0$ pour qu'elle ne soit pas nulle. L'intégration en $\theta$ impose que $l+l^{\prime}=0$.

Finalement on obtient :

$$
s \mathcal{U}=\sum_{n l q} \int_{S_{\mathrm{i}}}^{S_{\mathrm{e}}} \rho \mathrm{d} \rho \frac{\mu_{0} \pi^{2} n \omega}{i q k^{2}}\left[\left(H_{2} \dot{\dot{1}}+H_{3}\right)_{n l q}\left(u_{2}-u_{3}\right)_{-l-q}-\left(H_{2}-H_{3}\right)_{n l q}\left(u_{2}+u_{3}\right)_{-l-q}\right] \times \exp (i n \omega t) .
$$


En regroupant les termes $n l q$ et $-n-l-q$ comme dans le paragraphe 9 et en divisant par deux le résultat obtenu car le calcul effectué ici porte sur deux machines :

$$
s U=\frac{2 \pi^{2} \mu_{0} \omega}{k^{2}} \cdot \sum_{q>0} \sum_{n, l} \frac{n}{q} \Im m\left[\mathrm{e}^{i n \omega t} \int_{S_{\mathrm{i}}}^{S_{\mathrm{e}}}\left(H_{3} u_{3}^{*}-H_{2} u_{2}^{*}\right)_{n l q} \rho \mathrm{d} \rho\right] .
$$

12. Puissances à partir de la tension. - On peut facilement calculer la puissance active à partir du courant $s \mathfrak{J}(t)$ circulant dans le bobinage, de la tension à ses bornes et du nombre de bobines :

$$
\mathfrak{T}_{a}=m p \frac{\omega}{2 \pi} \int_{0}^{2 \pi / \omega} \mathrm{d} t \mathcal{U}(t) s J(t) .
$$

On reporte dans l'intégrale l'expression de :

$$
\mathfrak{J}=\sum_{n^{\prime} \in \mathbf{Z}} I_{n} \mathrm{e}^{i n^{\prime} \omega t}
$$

(avec $I_{n^{\prime}}=I_{-n^{\prime}}^{*}$ puisque $\mathfrak{J}$ est réel), ainsi que celle de $U$ (cf. (69)).

L'intégrale sur $t$ montre qu' il ne reste dans la sommation que les termes $n+n^{\prime}=0$ et l'on obtient finalement, en regroupant les termes $n l q$ et $-n-l-q$, une expression ressemblant beaucoup à (69) :

$$
\mathfrak{T}_{a}=\frac{2 \pi^{2} \mu_{0} \omega m p}{k^{2}} \sum_{q>0} \sum_{n, l} \frac{n}{q} J m\left[I_{-n} \int_{S_{\mathrm{i}}}^{S_{e}}\left(H_{3} u_{3}^{*}-H_{2} u_{2}^{*}\right)_{n l q} \rho \mathrm{d} \rho\right] .
$$

Remarquons que cette expression s'écrit aussi :

$$
\mathfrak{T}_{a}=2 m p \sum_{n \in \mathrm{N}} \operatorname{Re}\left(U_{n} I_{n}^{*}\right) .
$$

Nous disposons ainsi de deux expressions très différentes de la puissance active : l'une fait intervenir les champs au niveau du rotor, l'autre les champs sur l'ensemble du stator. Elles nous serviront en particulier à tester les calculs numériques.

Dans le cas où $\mathfrak{J}(t)$ est sinusoïdal, on peut aussi calculer la puissance réactive :

$$
\mathfrak{T}_{r}=m p \frac{\omega}{2 \pi} \int_{0}^{2 \pi / \omega} \mathrm{d} t u(t) s \mathfrak{s}\left(\omega t+\frac{\pi}{2}\right) .
$$

$J(t)$ ne comporte alors que deux composantes de Fourier $I_{1}$ et $I_{-1}=I_{1}^{*}$.

$$
\mathfrak{T}_{r}=\frac{2 \pi^{2} \mu_{0} \omega m p}{k^{2}} \sum_{q>0} \sum_{l} \frac{1}{q} \operatorname{Re}\left[I_{-1}\left(H_{3} u_{3}^{*}-H_{2} u_{2}^{*}\right)_{1 l q}\right] .
$$

De façon générale, on peut définir pour chaque harmonique $n$ :

$$
\mathfrak{T}_{r n}=m p \frac{n \omega}{2 \pi} \int_{0}^{2 \pi / n \omega} \mathrm{d} t \mathcal{U}_{n}(t) s \mathfrak{J}_{n}\left(n \omega t+\frac{\pi}{2}\right),
$$

puisque $\mathcal{U}_{n}=U_{n} \mathrm{e}^{i n \omega t}$, par analogie avec (7) et de même pour $J_{n}$.

En définissant la puissance réactive comme la somme sur tous les harmoniques, il vient :'

$$
\mathfrak{T}_{r}=\frac{2 \pi^{2} \mu_{0} \omega m p}{k^{2}} \sum_{q>0} \sum_{l} \frac{n}{q} \operatorname{Re}\left[I_{-n}\left(H_{3} u_{3}^{*}-H_{2} u_{2}^{*}\right)_{n l q}\right]
$$

ou encore

$$
\mathfrak{T}_{r}=2 m p \sum_{n \in \mathrm{N}} \mathfrak{J} m\left(U_{n} I_{n}^{*}\right)
$$

$\mathfrak{T}_{a}$ et $\mathfrak{T}_{r}$ sont donc les deux parties, réelle et imaginaire, d'une puissance complexe. 


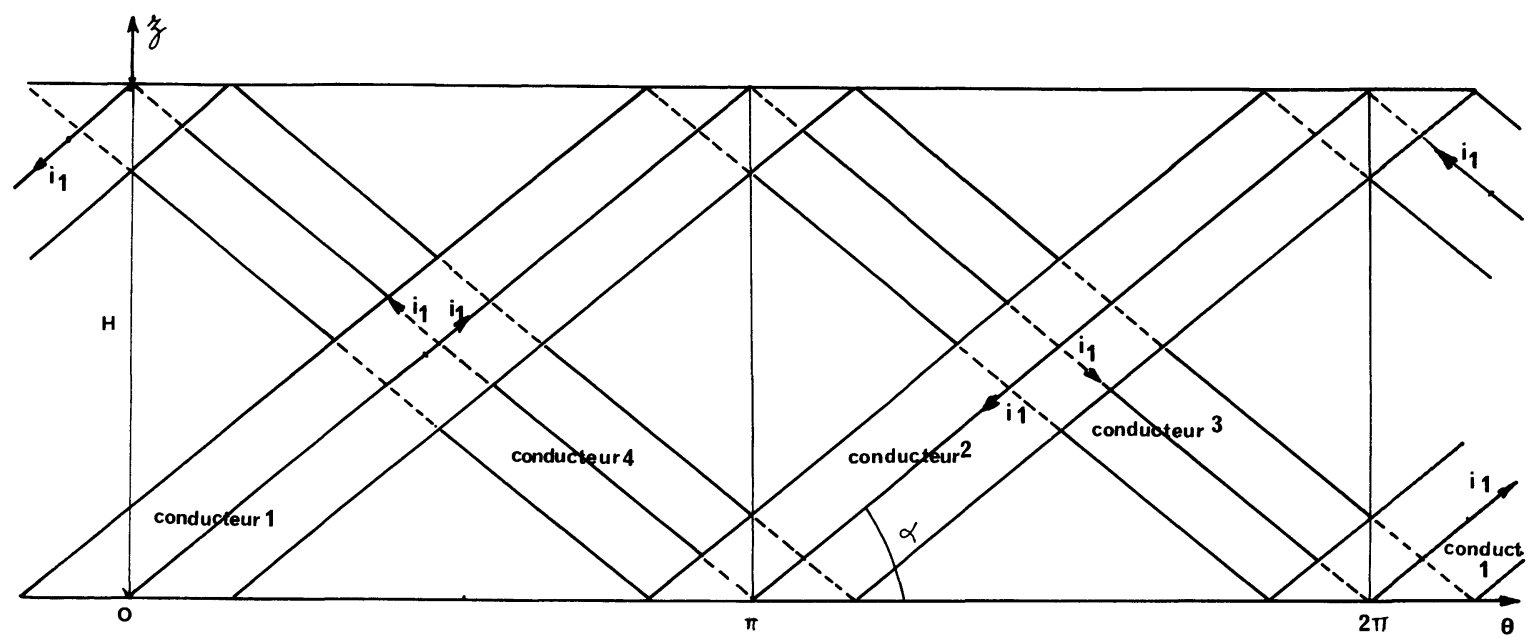

Fig. 4. - Développement d'une des trois bobines du stator.

[Development of one of the three stator coils.]

13. Expression des coefficients de Fourier d'un bobinage particulier. - On considère un bobinage triphasé dont la première phase est représentée en développé figure 4. Les conducteurs 1 et 2 sont placés sur une couche, les conducteurs 3 et 4 sur une autre plus extérieure, le contact est assuré entre les conducteurs 1 et 3 d'une part, 2 et 4 d'autre part. On admet que les courants ont les directions indiquées sur la figure; en particulier, on néglige toutes les composantes radiales qui peuvent exister au niveau des contacts. On suppose donc les conducteurs très plats : un bobinage épais sera formé par superposition de nombreuses couches.

Le vecteur u définissant la direction des courants $a$, par exemple pour le conducteur 1 , comme composantes :

$$
\begin{aligned}
& u_{1}(1)=u_{z}=\sin \alpha f(\theta, z), \\
& u_{2}(1)=u_{\rho}-i u_{\theta}=-i \cos \alpha f(\theta, z), \\
& u_{3}(1)=u_{\rho}+i u_{\theta}=i \cos \alpha f(\theta, z),
\end{aligned}
$$

avec $f(\theta, z)=1$ là où le conducteur 1 est présent et $f(\theta, z)=0$ ailleurs. Pour calculer la transformée de Fourier de u pour ce conducteur, il nous faut donc calculer celle de $f(\theta, z)$; on a, par une formule analogue à (8) :

$$
f_{l q}=\frac{\dot{k}}{(2 \pi)^{2}} \iint \mathrm{d} z \mathrm{~d} \theta \mathrm{e}^{-i q k z} \mathrm{e}^{-i l \theta} f(\theta, z)
$$

Intégrons tout d'abord en $\theta$, c'est-à-dire déplaçons le point d'intégration sur une horizontale $\mathbf{M}^{\prime} \mathbf{M}^{\prime \prime}(z=C$ te), puis intégrons en $z$ c'est-à-dire déplaçons le milieu $\mathbf{M}$ de l'horizontale sur toute la hauteur du conducteur (cf. Fig. 5). Il vient :

$$
f_{l q}=\frac{k}{(2 \pi)^{2}} \int_{\frac{\pi}{2 k}-\frac{H}{2}}^{\frac{\pi}{2 k}+\frac{H}{2}} \mathrm{~d} z_{\mathrm{M}} \mathrm{e}^{-i q k z_{\mathrm{M}}} \int_{\theta_{\mathrm{M}}-\frac{\pi}{6}}^{\theta_{\mathrm{M}}+\frac{\pi}{6}} \mathrm{~d} \theta \mathrm{e}^{-i l \theta},
$$

qui se calcule facilement.

On effectue le même type de calculs pour obtenir les expressions relatives aux conducteurs $2,3,4$ et au bobinage de la machine symétrique. Un bobinage réel étant composé pour moitié de conducteurs $1-2$ et pour moitié de conducteurs 3-4, la demi-somme de ces diverses expressions donne la transformée de Fourier d'une phase de l'ensemble des deux machines étudié :

$$
\begin{aligned}
& u_{1 l q}=\frac{\pi}{k H} \sin \alpha u_{0 l q}, \\
& u_{2 l q}=i \frac{q}{l} \cos \alpha u_{0 l q},
\end{aligned}
$$

où $l$ et $q$ sont impairs (les harmoniques avec $l$ ou $q$ pairs sont nuls).

$$
\begin{aligned}
q & =2 q^{\prime}+1, \\
u_{0 l q} & =\frac{4}{\pi^{2}} \cdot \frac{(-1)^{q^{\prime}}}{q^{2}-l^{2} \pi^{2} / k^{2} H^{2}} \sin \frac{l \pi}{6} \cos \frac{q k H}{2},
\end{aligned}
$$

(il faut choisir $k$ tel que $q k H \neq l \pi$ pour éviter tout problème d'indétermination de $u_{0 l q}$ ).

Comme nous l'avions annoncé, les harmoniques $l=0$ et $q=0$ sont bien nuls. 


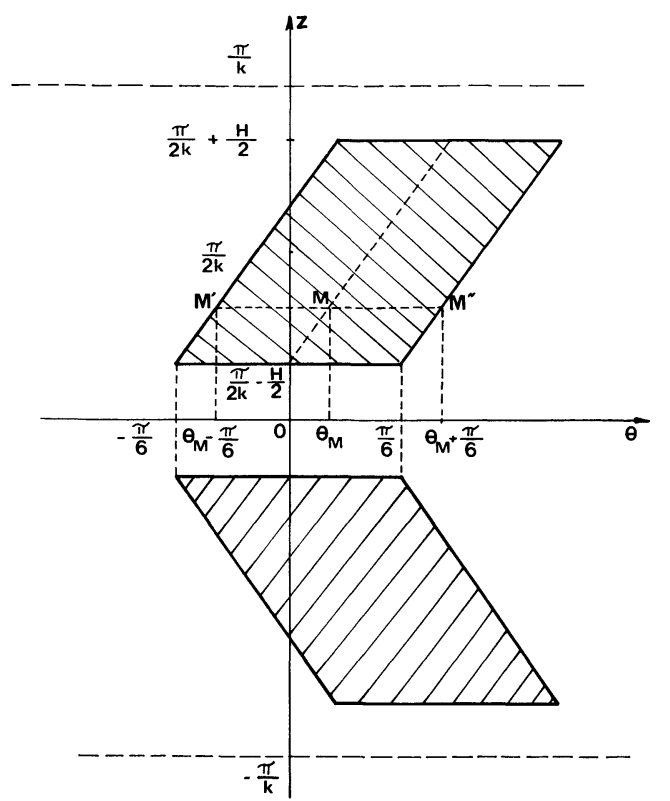

Fig. 5. - Domaine d'intégration pour le calcul des composantes de Fourier d'un conducteur du bobinage : les deux conducteurs représentés appartiennent à deux machines symétriques.

[The domain of integration for the calculus of the Fourier components of one coil : the two represented conductors belong to two symmetrical machines.]

14. Méthode de calcul. Exemple d'application. Le programme de calcul numérique comporte deux parties indépendantes :

- la détermination des coefficients de Fourier du bobinage,

- le calcul des champs et des grandeurs qui s'en déduisent.

La première partie nécessite le calcul d'expressions telles que (73) et (74). Il est évident que le bobinage décrit ici est d'un type particulièrement simple : il correspond à des maquettes que nous avons déjà construites (8).

La seconde consiste à résoudre le système linéaire $(61)+(62)$ ou $(61)+(63)$ suivant que la machine est entouree ou non d'une ceinture, les inconnues étant $A_{1}, A_{2}, A_{3}, G_{1}$ et $\widetilde{G}_{1}$.

Nous avons mis en place ce programme sur Univac 1110 et l'avons appliqué au cas d'une machine avec ceinture alimentée en courant sinusoïdal pur de pulsation $\omega$. La distance $\pi / k$ entre deux machines (cf. Fig. 2) était telle que $\pi / k H=3,1$ et $\pi / k R>10$ ce qui évitait que les champs générés par deux machines voisines interfèrent. La figure 6 donne à titre d'exemple la variation de la puissance active par unité de longueur de stator et du « $\cos \varphi$ » en fonction du glissement $\omega-\Omega$ dans le cas d'une géométrie particulière.

La figure 7 donne la variation de ces mêmes grandeurs en fonction de la longueur du stator pour une

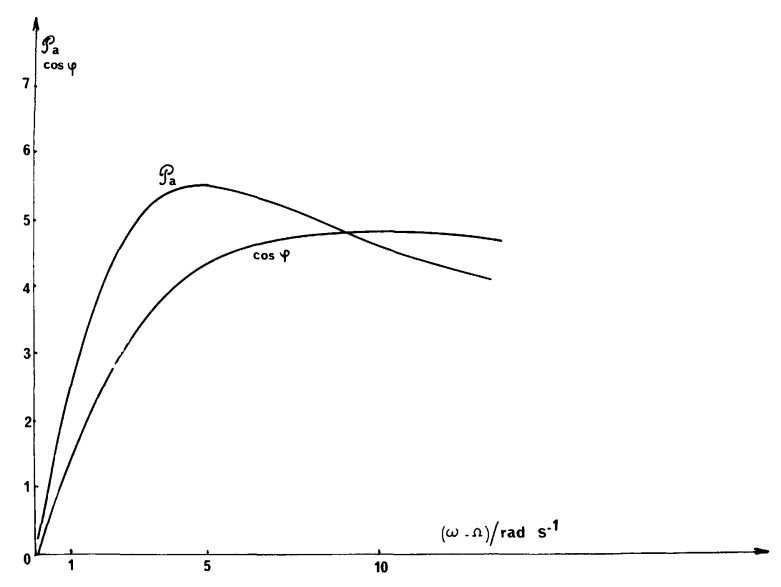

Fig. 6. - Variation de la puissance et du $\cos \varphi$ en fonction du glissement pour une machine telle que : $R=0,2, S_{\mathrm{i}}=$ $0,207, S_{\mathrm{e}}=0,261, F=0,265 ; H=1$ (en m).

[Variation of the power and of the $" \cos \varphi$ » as a function of the slip for a machine such that : $R=0.2, S_{\mathrm{i}}=0.207$, $S_{\mathrm{e}}=0.261, F=0.265 ; H=1(\mathrm{~m})$.]

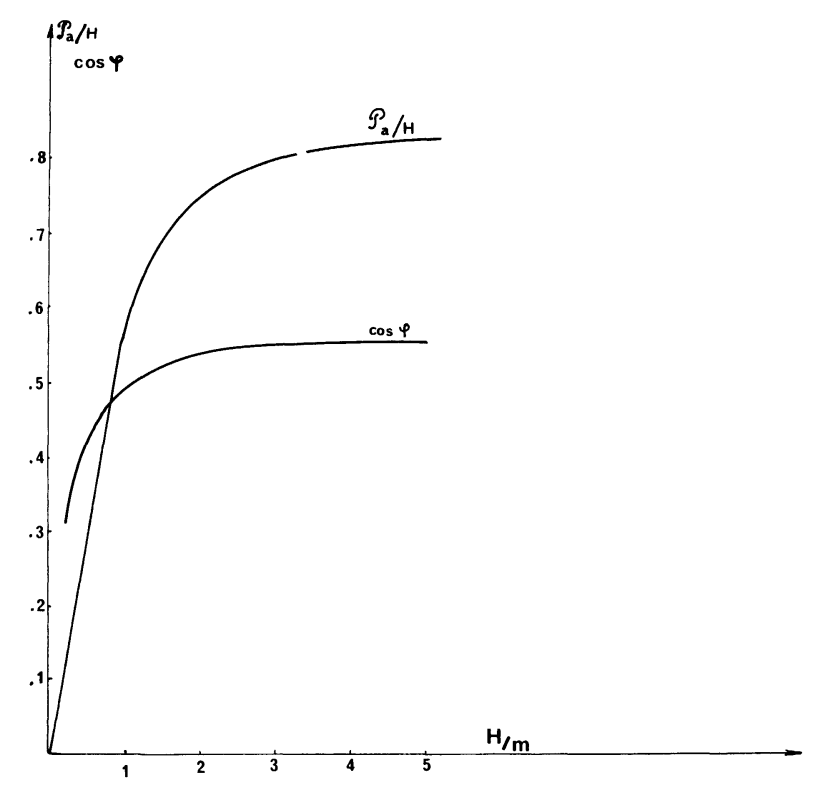

Fig. 7. - Variation de la puissance active et du $\cos \varphi$ en fonction de la longueur $H$ du stator pour une machine telle que : $R=0,15, S_{\mathrm{i}}=0,1689, S_{\mathrm{e}}=0,1690, F=0,1880$ (en $\mathrm{m}$ ).

[Variation of the active power and of the " $\cos \varphi$ " as a function of the length $H$ of the stator for a machine such that : $R=0.15, S_{\mathrm{i}}=0.1689, S_{\mathrm{e}}=0.1690, F=0.1880(\mathrm{~m})$.]

autre géométrie : l'intérêt des machines longues apparaît clairement.

Les calculs sont très rapides et nous avons constaté un accord remarquable entre les deux calculs de la puissance (par le couple et par la tension); on ne commence à constater un écart minime de $10^{-3}$ entre elles que pour les contributions des modes d'ordre très élevé, 19 ou 21 en $z$, lesquelles sont très faibles (environ $10^{-5}$ ). 
15. Conclusion. - Le calcul des champs magnétiques régnant dans les machines cylindriques sans fer à l'aide d'une décomposition en séries de Fourier à la fois spatiale (en $z$ et $\theta$ ) et temporelle a permis la première détermination de l'influence de la longueur finie du stator sur les performances de la machine. Le calcul numérique se révèle très rapide; il doit permettre l'étude de divers types de bobinage et par suite l'optimisation des structures.

\section{Bibliographie}

[1] Silvester, P., Chari, M., IEEE Trans. Power Appar. Syst. 89(1970) 1643.

[2] Ancelle, B., Coulomb, J. L., Masse, P., Meunier, G., SABonnadière, J. C., IEEE Trans. Magn. 15 (1979) 1671.

[3] Biedinger, J. M., Thèse de Docteur-Ingénieur, Compiègne (1980).

[4] Feliachi, M., Thèse de Docteur-Ingénieur, Paris (1981).
[5] Bleiss, C. A., Demonchy, M., Rioux, C., Electric machines and electromechanics 7 (1982) 351.

[6] Abramovitz, M., Stegun, J., Handbook of mathematical functions (Dover Publications, Inc. New York) 1965

[7] Angot, A., Compléments de mathématiques (Masson, Paris) 1972.

[8] Sultanem, F., Thèse de Docteur-Ingénieur, Paris (1977). 